\title{
Efficacy of post-procedural oral hydration volume on risk of contrast-induced acute kidney injury following primary percutaneous coronary intervention: study protocol for a randomized controlled trial
}

Feier Song ${ }^{1 \dagger}$, Guoli Sun ${ }^{2+}$, Jin Liu ${ }^{1 \dagger}$, Ji-yan Chen ${ }^{3 *}$, Yibo He ${ }^{1}$, Liwei Liư ${ }^{4}$, Yong Liu ${ }^{1 *}$ and the RESCIND group

\begin{abstract}
Background: Contrast-induced acute kidney injury (Cl-AKI) contributes toward unfavorable clinical outcomes. Oral hydration with water is inexpensive and it may be effective in the prevention of $\mathrm{Cl}$-AKI, but its efficacy among patients undergoing primary percutaneous coronary intervention $(\mathrm{PCl})$ remains unknown.

Methods/design: Our study is a secondary analysis on the database from the ATTEMPT study. We enrolled ST-elevation myocardial infarction (STEMI) patients undergoing primary PCI. Eligible patients received periprocedural aggressive (left ventricular end-diastolic pressure-guided) or routine ( $\leq 500 \mathrm{~mL}$ ) intravenous hydration with an isotonic solution $(0.9 \% \mathrm{NaCl})$ with randomization. The primary endpoint was $\mathrm{Cl}-\mathrm{AKl}$, defined as a $>25 \%$ or $0.5 \mathrm{mg} / \mathrm{dL}$ increase in serum creatinine from baseline during the first $48-72 \mathrm{~h}$ post-procedurally. All patients drank unrestricted amounts of fluids freely, the volume of which was recorded until $24 \mathrm{~h}$ following primary $\mathrm{PCl}$. Oral hydration volume/weight (OHV/W) ratios were calculated. The association between post-procedural oral hydration (quartiles) and Cl-AKI was assessed using multivariable analysis controlling for confounders, including intravenous hydration strategies.
\end{abstract}

Discussion: Our study determined the effects of post-procedural oral hydration on CI-AKI following primary $\mathrm{PCl}$, which is a potential strategy for Cl-AKI prevention among patients with STEMI at very high risk.

Trial registration: ClinicalTrials.gov, NCT02067195. Registered on 21 February 2014.

Keywords: Primary percutaneous coronary intervention, Contrast-induced acute kidney injury, Oral hydration, ST-elevation myocardial infarction, Contrast media

\section{Background}

Patients with ST-elevation myocardial infarction (STEMI) have a higher risk of contrast-induced acute kidney injury

\footnotetext{
*Correspondence: chenji_yandr@126.com; liuyong2099@126.com ${ }^{\dagger}$ Feier Song, Guoli Sun and Jin Liu contributed equally to this work. ${ }^{3}$ Department of Cardiology, Guangdong Cardiovascular Institute, Guangdong Provincial Key Laboratory of Coronary Heart Disease, Guangdong Provincial People's Hospital, Guangdong Academy of Medical Sciences, Guangzhou 510080, People's Republic of China

'Department of Cardiology, Guangdong Cardiovascular Institute, Guangdong Provincial Key Laboratory of Coronary Heart Disease, Guangdong Provincial People's Hospital, Guangdong Academy of Medical Sciences, Guangzhou 510080, Guangdong, People's Republic of China

Full list of author information is available at the end of the article
}

(CI-AKI) [1, 2]. CI-AKI is a potentially serious complication of angiographic procedures and can constitute up to $10 \%$ of hospital-acquired acute kidney injury [3]. Severe acute kidney injury requires renal replacement therapy and results in increased morbidity and mortality, prolonged hospitalization, and overall increased healthcare costs [4-6]. Of concern, $14.5 \%$ of the patients undergoing cardiac catheterization develop CI-AKI; reports have shown a 50\% incidence in high-risk patients [7, 8]. CI-AKI is associated with poorer outcomes, even in STEMI patients without impaired left ventricular ejection fraction (LVEF) and with normal renal function $[9,10]$. 
According to the 2018 ESC/EACTS guidelines on myocardial revascularization, prophylactic hydration is recommended in high-risk patients [11]. Previous metaanalyses focusing on different types of procedures (cardiac catheterization, enhanced computed tomography scans, angiography for peripheral vascular disease, and so on) have suggested that oral hydration may be as effective as intravenous hydration in preventing CI-AKI $[12,13]$. Conflicting results have arisen regarding whether oral hydration prevents CI-AKI. Previous studies have been conducted on relatively low-risk patients, including those subjects undergoing intravenous radiographic procedures or elective percutaneous coronary intervention (PCI). Optimal evidence for an oral hydration strategy has not been well established in a high-risk population such as STEMI patients undergoing primary PCI.

Given the data, we designed this study to evaluate the efficacy of post-procedural oral hydration for CI-AKI prevention in high-risk patients with STEMI undergoing primary PCI.

\section{Methods}

\section{Study design and population}

This is a secondary analysis of the Aggressive Hydration in Patients with ST-Elevation Myocardial Infarction undergoing Primary Percutaneous Coronary Intervention to Prevent Contrast-induced Nephropathy, the First Study for Reduction of Contrast-induced Nephropathy following Cardiac Catheterization (ATTEMPT RESCIND-1) study. Patients aged $\geq 18$ years with STEMI undergoing primary PCI who provided written informed consent are included from 15 academic medical centers in China. Patients are excluded for the following reasons: 1) contrast medium administration within the previous 7 days; 2) end-stage renal failure or renal transplantation; 3) inferior and/or right ventricle myocardial infarction combined with hypotension (defined as systolic pressure $\leq 90 \mathrm{mmHg}$ ) on admission; 4) pre-procedural renal insufficiency (history of chronic kidney disease or estimated glomerular filtration rate $\leq 60 \mathrm{~mL} / \mathrm{min} / 1.73 \mathrm{~m}^{2}$ calculated using the level-modified Modification of Diet in Renal Disease formula); 5) cardiogenic shock or New York Heart Association functional classification IV; 6) acute kidney injury defined as an absolute serum creatinine increase of $0.5 \mathrm{mg} / \mathrm{dL}$ from baseline obtained in the previous $24 \mathrm{~h}$; 7) lactation; 8) pregnancy; 9) malignant tumor or life expectancy $<1$ year; 10) allergy to contrast; 11) peri-procedural administration of nonsteroidal anti-inflammatory drugs, aminoglycosides, cyclosporine, or cisplatin in the previous $48 \mathrm{~h}$ or during the study period; 12) planned renal catheterization; or 13) heart valve surgery [14].

\section{Study protocol}

All eligible participants will receive peri-procedural intravenous hydration of different regimens. Oral hydration will begin at an unlimited rate until $24 \mathrm{~h}$ after the coronary procedure. By monitoring the fluid status, the rate and duration of oral intake, as well as the use of diuretics, are based on the clinical evaluation for heart function and at the discretion of the cardiologists.

Additionally, oral hydration volume/weight (OHV/W) ratios will be calculated. To determine the efficacy of oral hydration, we will collect data on the type of water taken, the volume per hour of water $(\mathrm{mL})$, the duration in the $24 \mathrm{~h}$ after the procedure, and any use of diuretics. Additionally, the urine output per hour of the patients will be observed. Hemodynamic data will be obtained in various clinical settings to assess volume status and guide medical therapy, including the administration of intravenous fluids.

Follow-up events will be carefully monitored and recorded by trained investigators through office visits and telephone interviews at 3,6,12,18, and 24 months after primary PCI. All data will be collected using standardized electronic case report forms. At the time of data entry, the data management team of Guangdong General Hospital will perform consistency checks and issue electronic data clarification forms to follow-up on discrepant data.

Data collected at enrolment will include baseline demographics, diagnosis, medical histories, clinical and laboratory parameters, concomitant drug treatment, and physical examination findings. Confounders such as age, sex, estimated glomerular filtration rate, LVEF, the use of an intra-aortic balloon pump, chronic heart failure, anemia, diabetes mellitus, and intravenous hydration will be measured to develop the logistic regression models. The primary outcome measure is CI-AKI, defined as a $>25 \%$ or $0.5 \mathrm{mg} / \mathrm{dL}$ increase in serum creatinine from baseline during the first $48-72 \mathrm{~h}$ post-procedure [15]. Secondary endpoints are the different definitions of CI-AKI, as well as persistent renal damage, major adverse cardiovascular events, major post-procedure in-hospital adverse clinical events, total hospitalization costs, and length of stay (Fig. 1).

All data from the study will be collected following approval by the corresponding ethics committees of the participating centers. An independent data monitoring committee will be responsible for the review of the ongoing safety of patients enrolled in the study (Additional file 1).

\section{Statistical analysis}

Baseline patient characteristics and information will be described as means \pm standard deviation or medians and interquartile ranges of descriptive statistics. Categorical 


\begin{tabular}{|c|c|c|c|c|c|c|c|c|}
\hline & \multicolumn{8}{|c|}{ STUDY PERIOD } \\
\hline \multirow[b]{2}{*}{ TIMEPOINT } & Enrolment & Allocation & \multicolumn{5}{|c|}{ Post-allocation } & \multirow{2}{*}{$\begin{array}{c}\text { Close-out } \\
\text { Year } 1\end{array}$} \\
\hline & Day $-1 \sim 0$ & Day 0 & $\begin{array}{c}\text { Day } \\
1\end{array}$ & $\begin{array}{c}\text { Day } \\
2\end{array}$ & $\begin{array}{c}\text { Day } \\
3\end{array}$ & $\begin{array}{c}\text { Day } \\
7\end{array}$ & $\begin{array}{c}\text { Day } \\
90\end{array}$ & \\
\hline \multicolumn{9}{|l|}{ ENROLMENT: } \\
\hline Eligibility screen & $\mathrm{X}$ & & & & & & & \\
\hline Informed consent & $\mathrm{X}$ & & & & & & & \\
\hline Allocation & & $\mathrm{X}$ & & & & & & \\
\hline \multicolumn{9}{|l|}{ INTERVENTIONS: } \\
\hline \multirow{2}{*}{$\begin{array}{r}\text { [General hydration] } \\
\text { [Aggressive } \\
\text { hydration] }\end{array}$} & & $\mathrm{X}$ & & & & & & \\
\hline & & $\mathrm{X}$ & & & & & & \\
\hline \multicolumn{9}{|l|}{ [Oral hydration] } \\
\hline \multicolumn{9}{|l|}{ ASSESSMENTS: } \\
\hline [Baseline variables] & $\mathrm{X}$ & $\mathrm{X}$ & & & & & & \\
\hline [CI-AKI] & & & $\mathrm{X}$ & $\mathrm{X}$ & $\mathrm{X}$ & & & \\
\hline [Mortality] & & & $\mathrm{X}$ & $\mathrm{X}$ & $\mathrm{X}$ & $\mathrm{X}$ & $\mathrm{x}$ & $\mathrm{X}$ \\
\hline
\end{tabular}

Fig. 1 The schedule of enrolment, interventions, and assessments. Cl-AKI contrast-induced acute kidney injury

variables will be presented as frequencies and percentages. $\mathrm{OHV} / \mathrm{W}$ ratios will be calculated and grouped according to the interquartile range. For continuous variables, one-way analysis of variance (ANOVA) will be conducted for normally distributed data, and the Kruskal-Wallis test used for non-normal distributions (presented as the median and interquartile range). Pearson's chi-square test or Fisher's exact test will be used, as appropriate, for categorical data.

The odds ratios of CI-AKI for quartiles with different $\mathrm{OHV} / \mathrm{W}$ ratios (cut-off values determined by the quartiles) will be estimated using univariate and multivariate logistic regression analyses. Multivariable logistic regression models have been developed to adjust for clinical characteristics (e.g., age, sex, estimated glomerular filtration rate, LVEF, the use of an intra-aortic balloon pump, chronic heart failure, anemia, diabetes mellitus, intravenous hydration), and the Mehran risk score will be calculated [16]. All variables that are univariately associated with this outcome measure will be entered as possible predictors in a multivariable logistic regression analysis. A two-tailed $p$ value $<0.05$ will be considered as statistically significant. The power calculation was based on our previous findings [17], and a CI-AKI incidence of $23 \%$ was estimated for the inadequate oral hydration group, while $11.5 \%$ was assumed for the adequate oral hydration group (50\% relative reduction). Using a two-sided chi-square test, a significance level of 0.05 , and a sample size of 280 each group, the power of the tests is $95.44 \%$.

All statistical analyses will be performed using SAS version 9.4 or later (SAS Institute, Cary, NC, USA) and $\mathrm{R}$ software (version 3.1.2; R Foundation for Statistical Computing, Vienna, Austria).

\section{Discussion}

Patients with STEMI are likely to present with hypotension or even shock, and it is often impossible to start renal prophylactic therapy, which is associated with an increased risk of CI-AKI [18]. Despite a considerable number of risk factors, including reduced LVEF, renal dysfunction, and diabetes mellitus in patients needing adequate hydration $[19,20]$, cardiologists in previous studies were primarily concerned with rapid revascularization for occluded culprit arteries instead of adequate pre-procedural hydration to prevent CI-AKI; this was related to a lack of information regarding baseline renal function and related medical history. Therefore, postprocedural oral hydration might be more suitable for CI-AKI prevention in patients with STEMI. 


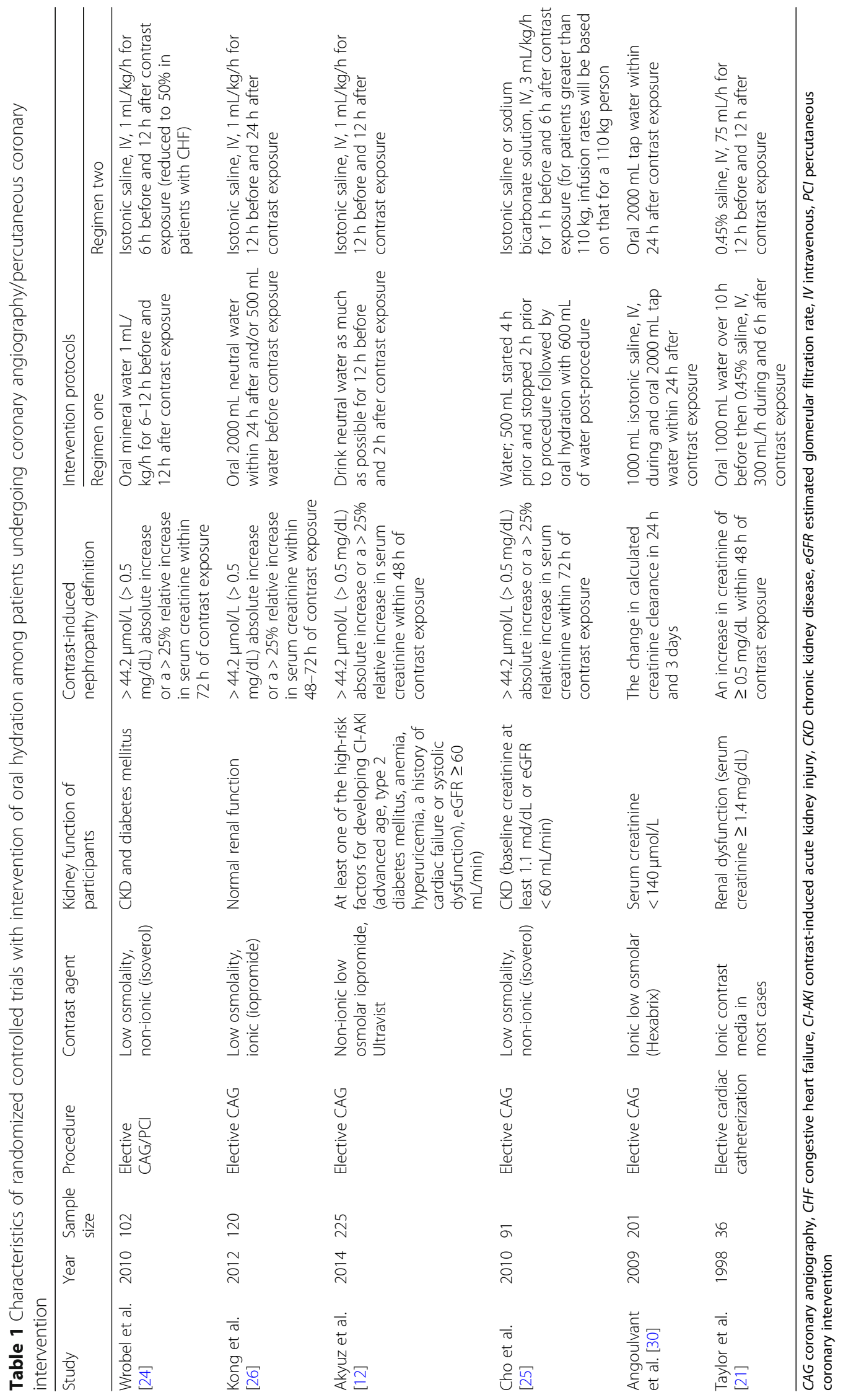


The first study, published in 1998, reported that oral hydration was as effective as intravenous hydration [21]. Two other studies applied oral hydration to the entire group with the aim of determining the incidence of CI-AKI [22] and comparing the efficacy of different oral volumes [23] where the incidence of CI-AKI could be reduced by oral hydration. The latest three studies reported similar results [24-26]. Four meta-analyses have been published so far, which included between four and eight randomized controlled trials (RCTs) [13, 27-29]. These studies also demonstrated no significant difference between oral fluid hydration and intravenous fluid hydration regimens in the prevention of CI-AKI, nor that the oral route was inferior. There have been heterogeneities in the reported incidence of CI-AKI among studies, due to differences in CI-AKI definition, study population, and imaging procedure (Table 1).

The above-mentioned studies were conducted on relatively low-risk patients, including those subjects undergoing intravenous radiographic procedures. The frequencies of risk factors were merely reported, and some RCTs excluded patients with chronic kidney disease, congestive heart failure, or systolic dysfunction, with a lower proportion of diabetic patients. Moreover, the oral hydration protocol varied greatly, with no two trials having a similar oral regimen.

It is reported that the incidence is $<2 \%$ in the general population but is up to $20-30 \%$ in high-risk populations with congestive heart failure, chronic kidney disease, diabetes mellitus, and anemia [3].

For in-patient settings or individuals who require emergent coronary angiography or radiological procedures with contrast exposures, intravenous hydration has been studied and used as first-line treatment for the prevention of CI-AKI [11]. However, there is no consensus regarding the mode of administration. In modern medicine, with an evolving number of diagnostic studies that depend on iodinated contrast along with an increasing number of complex high-risk patients, the costs of hospitalizations and nursing care are growing. Previous hydration strategies have not been investigated in STEMI patients. Therefore, oral hydration, which is considered safe and effective in low-risk patients, should be investigated in patients with STEMI undergoing primary PCI.

\section{Limitations}

Our current secondary analysis is subject to the following limitations. First, it was less sensitive than defined as a $>0.5 \mathrm{mg} / \mathrm{dL}$ increase in serum creatinine, because it recognized less selectively those patients with a higher risk of mortality and morbidity. Second, according to the ATTEMPT study, participants received either aggressive or routine intravenous hydration. Hemodilution can reduce serum creatinine, and cumulative daily fluid balance (input/output) directly affects the concentration (i.e., dilution) of serum creatinine. In our study, post-procedural daily fluid balance (input/output) was recorded to estimate the change in renal function to reduce the influence of hemodilution. Third, there were the inherent limitations of an observational study, including differences in oral hydration administration protocols regarding hydration rate, time, and total volume. Fourth, the fact that post-procedure serum creatinine measurements were not random but standardized at $48 \mathrm{~h}$ might suggest that delayed-onset elevation of serum creatinine $(>48 \mathrm{~h}$ ) could be overlooked. Finally, this was a secondary analysis, which is not able to conclude a causal relationship. On the basis of the above limitations, future large-sample, well-designed RCTs are required to confirm and update the findings of this secondary analysis. However, to the best of our knowledge, this is the first prospective, subanalysis to investigate the effect of oral hydration on the prevention of CI-AKI in high-risk patients undergoing primary $\mathrm{PCI}$.

Oral hydration has a practical value in daily life. It is easy to administer, allows better use of hospital resources due to shorter hospital stays, does not require intravascular cannulation, is less expensive, and is more comfortable for the patient. Our study will determine the association between post-procedural oral hydration and the decreasing incidence of CI-AKI following primary $\mathrm{PCI}$, as well as the risk factors among quartiles of OHV.

\section{Additional file}

Additional file 1: SPIRIT 2013 checklist: recommended items to address in a clinical trial protocol and related documents. (DOC $121 \mathrm{~kb}$ )

\section{Abbreviations}

Cl-AKI: Contrast-induced acute kidney injury; LVEF: Left ventricular ejection fraction; OHV/W: Oral hydration volume/weight; PCI: Percutaneous coronary intervention; RCT: Randomized controlled trial; STEMI: ST-elevation myocardial infarction

\section{Acknowledgments \\ The authors are solely responsible for the design and conduct of this study, all study analyses, and the drafting and editing of the paper and its final contents. Special thanks go to the project managers Mr. Yongquan Yang and Ms. Lingfen Chen for their considerable time and effort in the ATTEMPT study.}

\section{Funding}

This study was supported by grants from the Science and Technology Planning Project of Guangdong Province (no. 2014B070706010), the National Natural Science Foundation of China (no. 81670339), and the Guangdong General Hospital Clinical Research Fund and Guangdong Society of Interventional Cardiology Research Fund (no. 2014dzx02).

\section{Trial status}

The RESCIND-1 ATTEMPT trial (protocol version 2.0, 11 June 2014) enrolled the first participant on 1 July 2014 and expected to complete recruitment in 
December 2018. As of 24 October 2018, recruitment is ongoing with 555 patients randomized at 15 centers in China.

\section{Authors' contributions}

Conceptualization: FS. Project administration: JL, YL. Resources: YL, JC. Writing—original draft: FS. Writing—review and editing: FS, JL, GS, YH, LL. All authors read and approved the final manuscript.

\section{Ethics approval and consent to participate}

All participants provided written informed consent before enrolment. All data in the study will be collected following approval by the corresponding ethics committees of the participating centers. An independent data monitoring committee will be responsible for the review of the ongoing safety of patients enrolled in the study.

\section{Consent for publication}

Not applicable.

\section{Competing interests}

The authors declare that they have no competing interests.

\section{Publisher's Note}

Springer Nature remains neutral with regard to jurisdictional claims in published maps and institutional affiliations.

\section{Author details}

'Department of Cardiology, Guangdong Cardiovascular Institute, Guangdong Provincial Key Laboratory of Coronary Heart Disease, Guangdong Provincial People's Hospital, Guangdong Academy of Medical Sciences, Guangzhou 510080, Guangdong, People's Republic of China. ${ }^{2}$ Guangdong Provincial People's Hospital, School of Medicine, South China University of Technology, Guangzhou, People's Republic of China. ${ }^{3}$ Department of Cardiology, Guangdong Cardiovascular Institute, Guangdong Provincial Key Laboratory of Coronary Heart Disease, Guangdong Provincial People's Hospital, Guangdong Academy of Medical Sciences, Guangzhou 510080, People's Republic of China. ${ }^{4}$ The Second School of Clinical Medicine, Southern Medical University, Guangzhou, People's Republic of China.

\section{Received: 4 December 2018 Accepted: 7 May 2019}

\section{Published online: 27 May 2019}

\section{References}

1. Sadeghi HM, Stone GW, Grines $\mathrm{CL}$, et al. Impact of renal insufficiency in patients undergoing primary angioplasty for acute myocardial infarction. Circulation. 2003:108(22):2769-75.

2. Narula A, Mehran R, Weisz G, et al. Contrast-induced acute kidney injury after primary percutaneous coronary intervention: results from the HORIZONS-AMI substudy. Eur Heart J. 2014;35(23):1533-40.

3. Singri N, Ahya SN, Levin ML. Acute renal failure. JAMA. 2003;289(6):747-51.

4. McCullough PA, Wolyn R, Rocher LL, Levin RN, O'Neill WW. Acute renal failure after coronary intervention: incidence, risk factors, and relationship to mortality. Am J Med. 1997;103(5):368-75.

5. Tepel M, Aspelin P, Lameire N. Contrast-induced nephropathy: a clinical and evidence-based approach. Circulation. 2006;113(14):1799-806.

6. James MT, Ghali WA, Knudtson ML, et al. Associations between acute kidney injury and cardiovascular and renal outcomes after coronary angiography. Circulation. 2011;123(4):409-16.

7. Gupta R, Birnbaum Y, Uretsky BF. The renal patient with coronary artery disease: current concepts and dilemmas. J Am Coll Cardiol. 2004;44(7):1343-53.

8. Levy EM, Viscoli CM, Horwitz RI. The effect of acute renal failure on mortality. A cohort analysis. JAMA. 1996;275(19):1489-94.

9. Marenzi G, Lauri G, Assanelli E, et al. Contrast-induced nephropathy in patients undergoing primary angioplasty for acute myocardial infarction. J Am Coll Cardiol. 2004;44(9):1780-5.

10. Pyxaras SA, Sinagra G, Mangiacapra F, et al. Contrast-induced nephropathy in patients undergoing primary percutaneous coronary intervention without acute left ventricular ejection fraction impairment. Am J Cardiol. 2013;111(5): 684-8.

11. Neumann Franz-Josef,Sousa-Uva Miguel,Ahlsson Anders et al. 2018 ESC/EACTS Guidelines on myocardial revascularization. [J] Eur. Heart J. 2019;40:87-165.
12. Akyuz S, Karaca M, Kemaloglu OT, et al. Efficacy of oral hydration in the prevention of contrast-induced acute kidney injury in patients undergoing coronary angiography or intervention. Nephron Clin Pract. 2014;128(1-2): 95-100.

13. Agarwal SK, Mohareb S, Patel A, et al. Systematic oral hydration with water is similar to parenteral hydration for prevention of contrast-induced nephropathy: an updated meta-analysis of randomised clinical data. Open Heart. 2015:2(1):e000317.

14. Liu Y, Chen JY, Huo Y, et al. Aggressive hydration in patients with STelevation myocardial infarction undergoing primary percutaneous coronary intervention to prevent contrast-induced nephropathy (ATTEMPT): study design and protocol for the randomized, controlled trial, the ATTEMPT, RESCIND 1 (first study for reduction of contrast-induced nephropathy following cardiac catheterization) trial. Am Heart J. 2016;172:88-95.

15. Briguori C, Visconti G, Rivera NV, et al. Cystatin C and contrast-induced acute kidney injury. Circulation. 2010;121(19):2117-22.

16. Mehran R, Aymong ED, Nikolsky E, et al. A simple risk score for prediction of contrast-induced nephropathy after percutaneous coronary intervention: development and initial validation. J Am Coll Cardiol. 2004:44(7):1393-9.

17. Tan N, Liu Y, Chen JY, et al. Use of the contrast volume or grams of iodineto-creatinine clearance ratio to predict mortality after percutaneous coronary intervention. Am Heart J. 2013;165(4):600-8.

18. Zoungas S, Ninomiya T, Huxley R, et al. Systematic review: sodium bicarbonate treatment regimens for the prevention of contrast-induced nephropathy. Ann Intern Med. 2009;151(9):631-8.

19. Sgura FA, Bertelli $L$, Monopoli $D$, et al. Mehran contrast-induced nephropathy risk score predicts short- and long-term clinical outcomes in patients with ST-elevation-myocardial infarction. Circ Cardiovasc Interv. 2010;3(5):491-8

20. Oduncu V, Erkol A, Karabay CY, et al. Relation of the severity of contrast induced nephropathy to SYNTAX score and long-term prognosis in patients treated with primary percutaneous coronary intervention. Int J Cardiol. 2013; 168(4):3480-5

21. Taylor AJ, Hotchkiss D, Morse RW, McCabe J. PREPARED: preparation for angiography in renal dysfunction: a randomized trial of inpatient vs outpatient hydration protocols for cardiac catheterization in mild-tomoderate renal dysfunction. Chest. 1998;114(6):1570-4.

22. Garcia-Ruiz C, Martinez-Vea A, Sempere T, et al. Low risk of contrast nephropathy in high-risk patients undergoing spiral computed tomography angiography with the contrast medium iopromide and prophylactic oral hydratation. Clin Nephrol. 2004;61(3):170-6.

23. Yoshikawa D, Isobe S, Sato K, et al. Importance of oral fluid intake after coronary computed tomography angiography: an observational study. Eur J Radiol. 2011;77(1):118-22.

24. Wrobel W, Sinkiewicz W, Gordon M, Wozniak-Wisniewska A. Oral versus intravenous hydration and renal function in diabetic patients undergoing percutaneous coronary interventions. Kardiol Pol. 2010;68(9):1015-20.

25. Cho R, Javed N, Traub D, et al. Oral hydration and alkalinization is noninferior to intravenous therapy for prevention of contrast-induced nephropathy in patients with chronic kidney disease. J Interv Cardiol. 2010; 23(5):460-6

26. Kong DG, Hou YF, Ma LL, Yao DK, Wang LX. Comparison of oral and intravenous hydration strategies for the prevention of contrast-induced nephropathy in patients undergoing coronary angiography or angioplasty: a randomized clinical trial. Acta Cardiol. 2012;67(5):565-9.

27. Cheungpasitporn W, Thongprayoon C, Brabec BA, et al. Oral hydration for prevention of contrast-induced acute kidney injury in elective radiological procedures: a systematic review and meta-analysis of randomized controlled trials. N Am J Med Sci. 2014;6(12):618-24.

28. Hiremath S, Akbari A, Shabana W, Fergusson DA, Knoll GA. Prevention of contrast-induced acute kidney injury: is simple oral hydration similar to intravenous? A systematic review of the evidence. PLoS One. 2013;8(3):e60009.

29. Zhang W, Zhang J, Yang B, et al. Effectiveness of oral hydration in preventing contrast-induced acute kidney injury in patients undergoing coronary angiography or intervention: a pairwise and network metaanalysis. Coron Artery Dis. 2018;29(4):286-93.

30. Angoulvant $D$, Cucherat $M$, Rioufol $G$ et al. Preventing acute decrease in renal function induced by coronary angiography (PRECORD): a prospective randomized trial. Arch Cardiovasc Dis. 2009;102(11):761-767. 